\title{
Performance Analysis of Adaptive Noise Canceller in Digital Filter for Human Voice Separation
}

\author{
${ }^{* 1}$ Dr. Swati S. Godbole, ${ }^{2}$ Dr. Sanjay B. Pokle \\ ${ }^{1}$ Department of Electronics Engineering, Shri Ramdeobaba College of Engineering \& Management, Nagpur, India \\ ${ }^{2}$ Department of Electronics \& Comm. Engineering, Shri Ramdeobaba College of Engineering \& Management, \\ Nagpur, India \\ Email: godboless@rknec.edu,poklesb@rknec.edu
}

Received: $20^{\text {th }}$ September 2018, Accepted: $11^{\text {th }}$ October 2018, Published: $31^{\text {st }}$ October 2018

\begin{abstract}
This Paper involves the performance analysis of Adaptive Noise Cancellation system (ANC) and its noise cancellation application by taking more realistic noises. Noise cancellation is a common occurrence in today's telecommunication environment. The main purpose of this research is to eliminate unwarranted noise that exists in incoming signal, which makes it difficult to understand. Adaptive Noise Cancellation is an alternative technique of estimating signals corrupted by additive noise or interference.

This paper examines adaptive filtering, employing discrete signal processing in MATLAB. Simulation was utilized by using MATLAB software to eliminate the noise. The strategies \& design methodologies of Adaptive Noise Canceller using the least mean square (LMS) algorithm is considered in this paper. Using the LMS algorithm, adaptive noise canceller has been implemented on MATLAB successfully by considering more realistic noises for suitable filter order and convergence factor. The results are analyzed for different input noises originating from refrigerators, vaccum cleaners, hair dryers, washing machines as reference signals and speech signal corrupted by these noises as primary signal. These signals are tested and analyzed and the filter performance of these noises is measured in terms of signal to noise ratio (SNR) improvement. From the experimentation it is clear that signal to noise ratio of primary signal earlier it was $3.468 \mathrm{db}$ which gets substantially improved by $49.921 \mathrm{~dB}$. It has also been observed that for filter order $\mathrm{N}=50$ and for convergence factor $\mu=0.03$, the voice signal can still be recovered perfectly.
\end{abstract}

\section{Keywords}

Adaptive Noise Canceller (ANC), Least Mean Square (LMS) Algorithm, Signal to Noise Ratio (SNR)

\section{Introduction}

Acoustic problems have been in focus in the wake of technological growth that gave rise to undesirable noise emerge from different sources like household appliances to heavy machineries, sound systems etc. Such difficulties can sometimes trouble the neighbors next door.

This method employ two different inputs, one is primary input that is information corrupted by noise \& second reference input that is only noise [1].

For the processing of non-stationary signals adaptive filters are widely used \& they are applied in different facets of communication. It consist of two main blocks, one is digital filter \& second is adaptive evaluation. Corrupted information is effectively process by digital filter whereas algorithm is used for adaptation purpose by altering the coefficient \& tries to optimize the desired weights [2-3].

The LMS algorithm [1] is undoubtedly the most popular algorithm for adapting the impulse response $W=\left[W^{0} \ldots \ldots . W^{N-1}\right]$ of an FIR filter so as to minimize the mean-square error (MSE) between its output signal $y(n)$ and a desired response signal $d(n)$. LMS algorithm is summarized as follows:

Determine $M, \mu$ and $w(0)$, at time $n=0$.

1. Compute the adaptive filter output

$$
y(n)=\sum_{i=0}^{M-1} w i(n) \times u(n-i)
$$

2. Compute the error signal

$$
e(n)=d(n)-y(n)
$$

3. Update the adaptive weight vector from $w(n)$ to $w(n+1)$ by using the LMS algorithm $w(n+1)=w(n)+2 \mu e(n) u(n)$

The LMS algorithm is a non-vanishing step size version of a stochastic gradient algorithm. It is popular to a large extent due to its computational similarity. Also, the functionally is quite simple to understand and the algorithm appears to be fairly compatible against implementation errors. 


\section{Proposed Approach: Adaptive Noise Cancellation}

This work aims to enhance the performance of adaptive filter by improving its signal to noise ratio. In adaptive noise cancellation, the adaptive filter is usually modelled as a transversal FIR filter structure. The concept of ANC filter in the block diagram is shown in Figure 1.

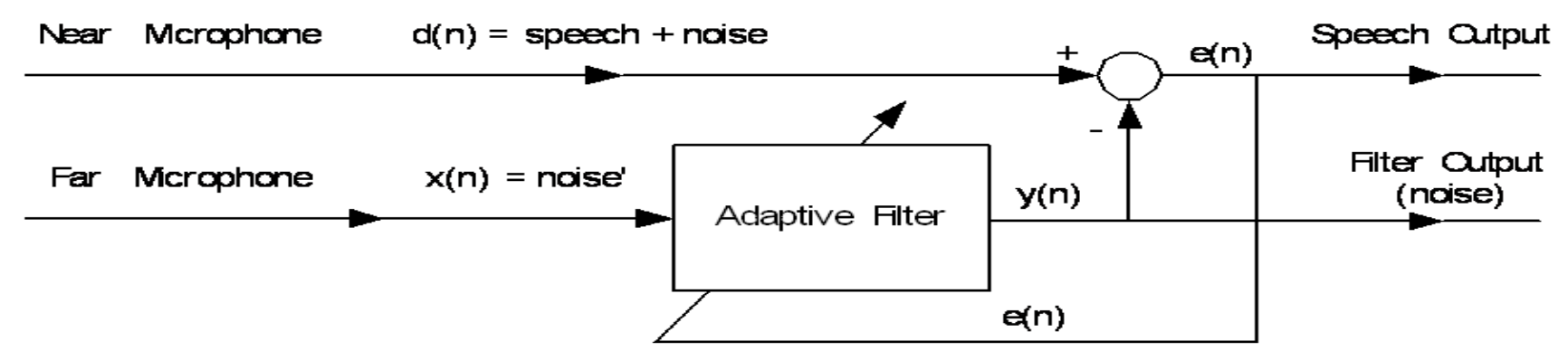

Figure 1: Basic Concept of Adaptive Noise Canceling



Figure 2: FIR LMS Filter Realization

ANC system operates like an automatic control system shown in figure 1. This system is based on the processing of two input signals, the primary signal i.e. information speech signal corrupted by noise and the noise signal called reference signal. This signal has to be match to the noise present in the primary input. The basic idea is to use adaptive filter applied to the reference input and to subtract its output from the primary input. LMS algorithm is used to update the filter coefficient in such a way that the noise is cancelled out from the corrupt speech signal and a clean speech signal is received. So, when the filter is optimized, the output will be a clean speech information signal as shown in figure 2. Amongst others LMS algorithm can converge quickly and has good noise suppression ability [1-2].

In this research, different parameters are analyzed by considering order of the filter, its convergence speed \& optimal hardware utilization. Figure 1 shows the block diagram of the adaptive noise cancellation system. The basic concept of adaptive noise cancellation is to process signal from two sensors and to reduce the level of the undesired noise with adaptive filtering techniques. The signal is transmitted over a channel to a sensor that receives the signal in addition to an uncorrelated noise, $n$. The combined signal and noise, $d+n$, form the 'primary input' to the canceller. A second sensor receives a noise $n$, which is uncorrelated with the signal but correlated in some unknown way with the noise $n$. This sensor provides the 'reference input' to the canceller. The noise $n$ ' generates from the same source as $n$ but can be modified by the environment. The noise $n$, is filtered to produce an output $y$ that is a close replica of $n$ i.e. the adaptive filter's output $y$ is adapted to the noise $n$.

Now, the obtained output $y$ is subtracted from the primary input to produce the system output which would be noise free. Subsequently, the filter output could now be subtracted from the primary input hence, producing an output which would be nothing but the signal alone.

The resulting signal is called as an error signal $e$, and is the output of the system. The resulting error signal would be only the desired portion of the primary signal. This error signal is fed back to the adaptive filter and is used to update the adaptive filter's coefficients until the overall output becomes $\mathrm{e}=d$. When this happens, the adaptation process is finished, and the 
error signal approaches the desired signal $d$ hence, the overall output is this error signal and not the adaptive filter's output $y$. If $d$ is uncorrelated with $n$, then the strategy is to minimize $E\left(e^{2}\right)$.

Thus in the system the reference input is processed by an adaptive filter that automatically adjusts its own impulse response through a least squares algorithm such as LMS that responds to an error signal dependent among other things, on the filters output[8-10]. Thus with the proper algorithm the filter can operate under varying conditions and can readjust itself continuously to minimize the error signal[11- 16].

In year 1960, work related to ANC was carried out by Howells and Applebaum and their colleagues at the GE Company. A system was designed to cancel antenna side lobe by using reference input obtained from auxiliary antenna [2].

ANC technique was developed at Stanford University in 1959 by Widrow and Hoff. In the beginning very few people were interested in adaptive filter and its high order adaptive processing. During same period, adaptive filters were under development at Great Britain by D. Gabor and their colleagues [3].

In mid sixties, the development of adaptive filters gained momentum. Many research papers were published related to adaptive controlling, filtering $\&$ signal processing. In digital communication, Lucky at the Bell Laboratories have worked on many commercial applications of adaptive filter during in early 1960 [4-5]. ANC technique was developed at Stanford University in 1959, with an idea to cancel $60 \mathrm{~Hz}$ of noise in output of electrocardiographic amplifier.

Since 1965, adaptive noise canceling has successfully been deployed to solve a various additional problems, including other aspects of electrocardiography, the removal of periodic \& aperiodic interference found, cancelling the donor ECG, in adaptive speech enhancement and channel equalization and echo cancelling over extended telephone transmission [6- 10].

\section{Non-Real Time Implementation of ANC System for Non-Stationary Signals}

The result shows improved performance of Adaptive Noise Cancellation system in MATLAB by considering more realistic noise signals. The designed system uses the LMS algorithm through a MATLAB program. The input signals such as primary and reference signals are recorded. These recorded signals are stored as .wav files and the .wav files are imported into the code. The intermediate signals such as speech corrupted with noise and output signals along with error signal and signals obtained after noise cancellation can be stored as .wav files. Thus, the audio of the voice signals can be literally heard, that helps immensely in judgments with respect to the results obtained.

Proper step size is selected to determine effective rate of convergence. For this purpose, realistic signals recorded \& used as .WAV file. The same is effectively created using Graphical user interface for better understanding.

Output waveforms of the system are plotted in the figures below.

Description Of The Simulation Setup:

\begin{tabular}{|l|l|l|}
\hline Sr. No. & Parameters & Used \\
\hline 1 & Order of filter & 50,30 \\
\hline 2 & Sampling rate & $11.025 \mathrm{KHz}, 44.1 \mathrm{KHz}$ \\
\hline 3 & Convergence Factor & 0.03 \\
\hline
\end{tabular}

Table 1: Analysis of Proposed Parameters and its Performance

The optimal parameters that are considered for effective implementation of ANC are the length of the filter taken to be 50 and 30 taps. The sampling frequency is taken to be $11.025 \mathrm{KHz}$ while the convergence factor used for the LMS algorithm is 0.03. Thus, various parameters considered for effective noise cancellation were successfully tested as per the proposed design.

It is evident that the designed ANC system uses the LMS algorithm through a MATLAB program. The input signals such as primary and reference signals are recorded and saved as .WAV files. The output of ANC i.e. error signal after cancellation of noise is generated and saved as.WAV file.

The signal processing toolbox has in-built functions for almost all signal processing applications. The toolbox enhances the efficiency of the code as these functions can be called wherever necessary instead of writing separate sub-routines.

For experimental setup, its prerequisites are:

1. Both recorded signals are applied to ANC for execution \& simulation.

2. The input signals was chosen at $10 \mathrm{sec}, 15 \mathrm{sec}, 20 \mathrm{sec}$.

Following is a summary of Widrow's LMS algorithm:

1. Find the values of $N, \mu, w(0)$, where $N$, is no. of taps, $\mu$ convergence factor, $\& w(0)=0$ i.e. initial weight

2. Calculate the output of filter

3. Find error output 
4. Adaptation of weights by applying error signal in feedback. While using error in feedback, weights are corrected simultaneously to find minimum mean square error.

5. Again iterate through the adaptive process until the next output is obtained.

\section{Simulation and Test of the Proposed Design}

\section{Experiment 1: Signal Length - 15 sec}

Experiment 1 is performed with following two signals.

Primary Signal : Speech Signal corrupted by Vaccum Cleaner Noise recorded at t2 (it's in a female's voice)

Reference Signal : Vaccum Cleaner Noise recorded at t1

Observations \& Result

The primary signal is generated in laboratory by mixing two audio signals, i.e. female speech as original signal contaminated the vacuum cleaner noise signal one by one as mentioned above, and then adaptive noise cancellation system filters them by self adjusting the phase and amplitude of a signal.

The figure 3 below shows three panels. The top panel is the reference signal vacuum cleaner noise recorded at $t$. The middle panel shows speech signal contaminated by vacuum cleaner noise recorded at t2. The bottom panel shows the adaptively filtered output speech signal. It is observed that the noise dies away over a few tenths of a second.

\begin{tabular}{|l|l|l|l|}
\hline Sr. No & Inputs & $\begin{array}{l}\text { SNR of Primary } \\
\text { Signal }(\mathrm{dB})\end{array}$ & $\begin{array}{l}\text { SNR of Filtered } \\
\text { Signal }(\mathrm{dB})\end{array}$ \\
\hline 1 & $\begin{array}{l}\text { Primary Signal }: \text { speech signal corrupted by vacuum } \\
\text { cleaner noise recorded at t2 }(15 \mathrm{sec}) \\
\text { Reference Signal: vacuum cleaner noise recorded at t1 } \\
\text { (Figure 3) }\end{array}$ & 2.6488 & 37.1842 \\
\hline
\end{tabular}

Table 2: Signals to Noise Ratio Analysis

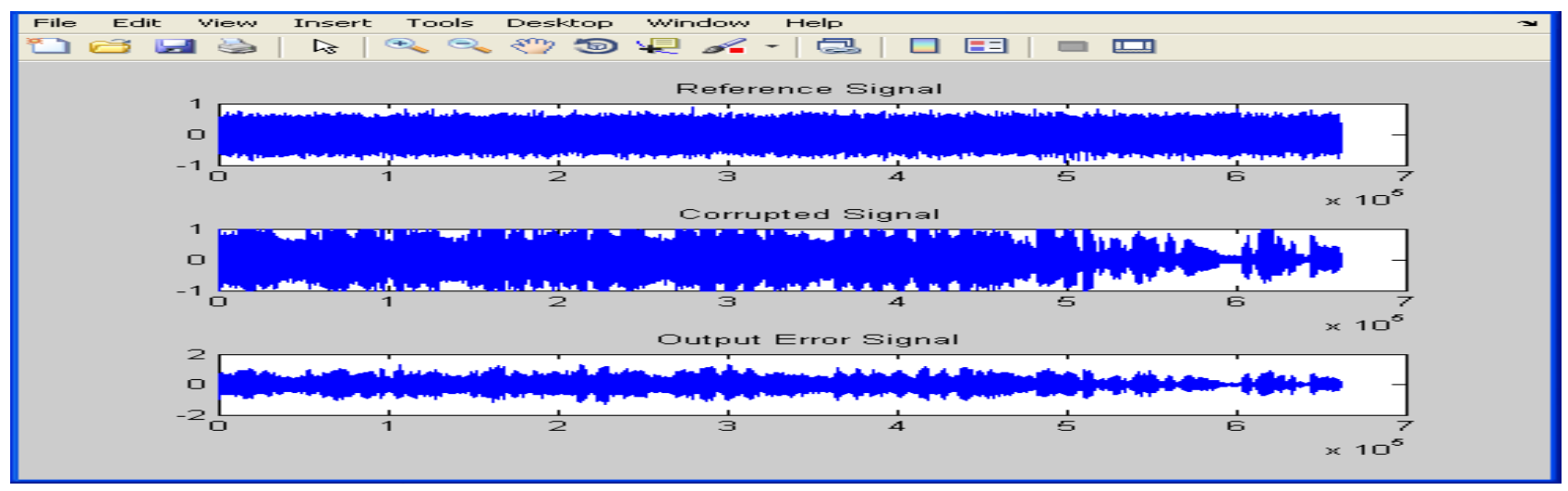

Figure 3: Filtered Speech Signal from Corrupted Vaccum Cleaner Noise

It can be seen from Table II, when the designed system is tested with vaccum cleaner noise, it shows significant SNR improvement as shown above. The ANC system performance is further tested by making variations in the amplitude and frequency of the undesired input vaccum cleaner noise signal that reflects improved SNR of the system.

\section{Experiment 2: Signal length - $1.5 \mathrm{sec}$}

Experiment 2 is performed with following two signals.

Primary Signal : Speech Signal corrupted by Vaccum Cleaner Noise recorded at t2

Reference Signal : vaccum cleaner noise with low speech recorded at t1

\section{Observations \& Result:}

The second experiment is carried out on the primary signal i.e. female speech signal as the desired signal contaminated with the vaccum cleaner noise recorded at $t 2$ and the reference signal i.e. undesired vaccum cleaner noise with low speech recorded at $\mathrm{t} 1$ 
While implementation of ANC system, it's observed that the output error signal E converged to the desired female speech signal, depicting the vaccum cleaner noise signal being cancelled gradually. Figure 4 shows the primary signal (female speech signal + vacuum cleaner noise) and the desired filtered output (female speech signal) after the adaptation process.

\begin{tabular}{|l|l|l|l|}
\hline S. No. & Inputs & $\begin{array}{l}\text { SNR of Primary Signal } \\
(\mathrm{dB})\end{array}$ & $\begin{array}{l}\text { SNR of Filtered } \\
\text { Signal }(\mathrm{dB})\end{array}$ \\
\hline 1 & $\begin{array}{l}\text { Reference Signal: vaccum cleaner noise with low speech } \\
\text { recorded at t1 }(1.5 \mathrm{sec}) \\
\begin{array}{l}\text { Primary Signal }: \text { speech signal corrupted by vaccum } \\
\text { cleaner noise recorded at t2 }(1.5 \mathrm{sec})\end{array}\end{array}$ & 3.4687 & 49.9213 \\
\hline
\end{tabular}

Table 3: SNR Analysis

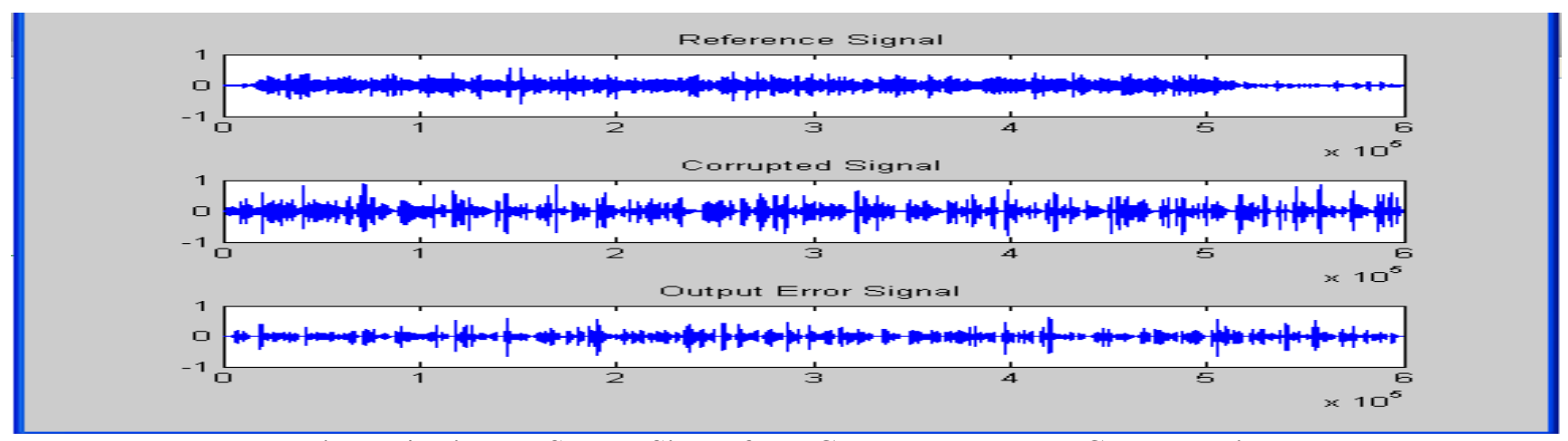

3. Experiment 3:

Figure 4: Filtered Speech Signal from Corrupted Vaccum Cleaner Noise

Primary Signal: speech signal + Gaussian noise

Reference Signal Gaussian noise:

$\mathrm{x}=\mathrm{x} 1+0.1 * \operatorname{randn}(\operatorname{size}(\mathrm{x} 1))$

$\mathrm{x} 1=$ wavread('300tone.wav');

\begin{tabular}{|l|l|l|}
\hline Sr. No. & Parameters & Used \\
\hline 1 & Order of filter & 30 \\
\hline 2 & Sampling rate & $44.1 \mathrm{KHz}$ \\
\hline 3 & Convergence Factor & 0.05 \\
\hline
\end{tabular}

Table 4: Analysis of Proposed Parameters and its Performance

For experiment 3, parameters that are taken for ANC are the length of the filter $=30$. The sampling frequency is taken as 44.1 $\mathrm{KHz}$ while the convergence factor used for the LMS algorithm is 0.05 .

Observations \& Result:

\begin{tabular}{|l|l|l|c|}
\hline S. No. & Inputs & $\begin{array}{l}\text { SNR of Primary } \\
\text { Signal }(\mathrm{dB})\end{array}$ & $\begin{array}{l}\text { SNR of Filtered } \\
\text { Signal }(\mathrm{dB})\end{array}$ \\
\hline 1 & $\begin{array}{l}\left.\text { Reference Signal: } \mathrm{x}=\mathrm{x} 1+0.1^{*} \text { randn(size }(\mathrm{x} 1)\right) \text { Primary Signal } \\
: \text { recorded female speech signal }+ \text { Gaussian noise }\end{array}$ & -1.3353 & 30.0301 \\
\hline
\end{tabular}

Table 5: SNR Analysis (Time_in_sec = 27.4460) 


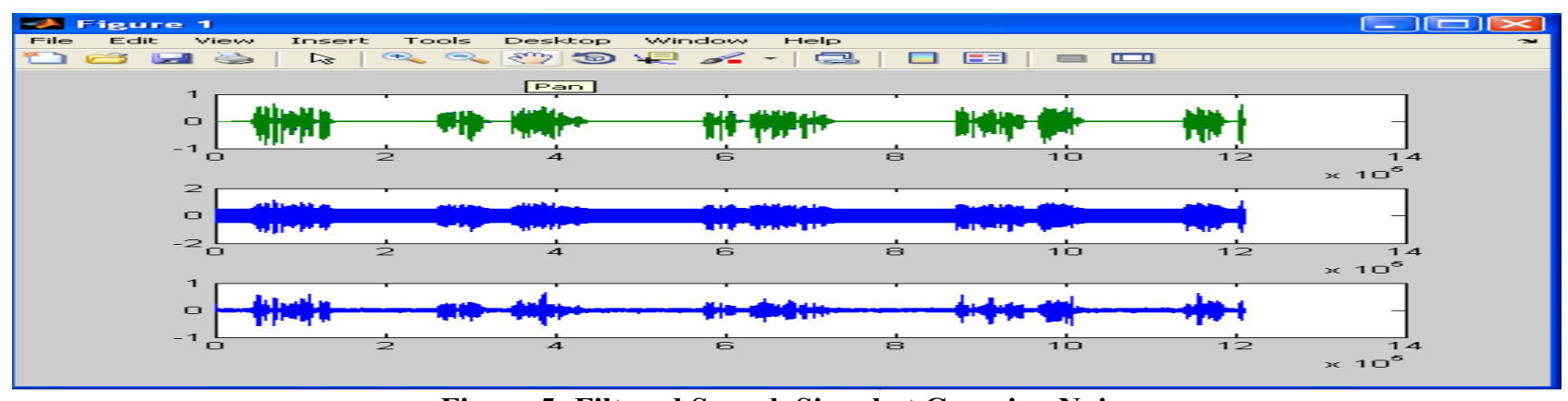

4. Experiment 4:

Figure 5: Filtered Speech Signal at Gaussian Noise

A) Inputs : Reference Signal : dogs barking noise

Primary Signal : speech signal + dogs barking noise

\section{Observations:}

\begin{tabular}{|l|l|l|l|}
\hline S.No. & Inputs & $\begin{array}{l}\text { SNR of Primary Signal } \\
(\mathrm{dB})\end{array}$ & $\begin{array}{l}\text { SNR of Filtered Signal } \\
(\mathrm{dB})\end{array}$ \\
\hline $\mathbf{1}$ & $\begin{array}{l}\text { Reference Signal: dogs barking noise } \\
\text { Primary Signal }: \text { recorded female speech } \\
\text { signal + dogs barking noise }\end{array}$ & $0.2090 \mathrm{~dB}$ & $-0.4564 \mathrm{~dB}$ \\
\hline
\end{tabular}

Table 6: SNR Analysis (Signal Length:Time_in_sec = 1 sec)

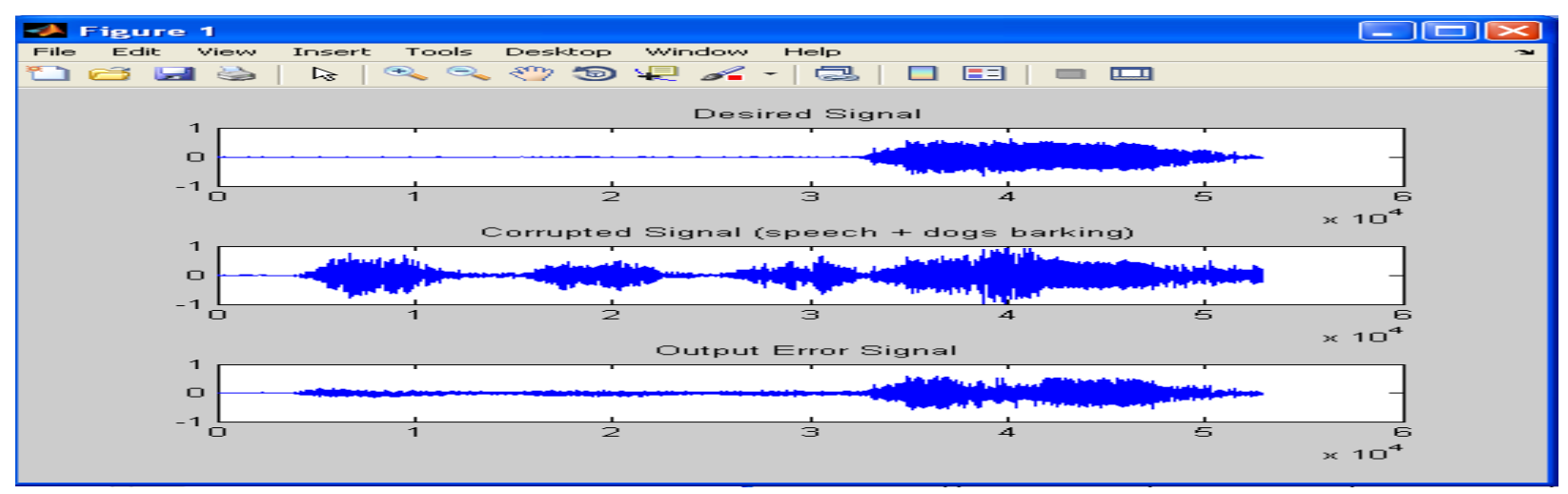

Figure 6: Filtered Speech Signal from Corrupted Dogs Barking Noise

B) Inputs : Reference Signal : washing machine noise

Primary Signal : speech signal + washing machine

noise

Observations:

\begin{tabular}{|l|l|l|l|}
\hline S.No. & Inputs & $\begin{array}{l}\text { SNR of Primary Signal } \\
(\mathrm{dB})\end{array}$ & $\begin{array}{l}\text { SNR of Filtered } \\
\text { Signal }(\mathrm{dB})\end{array}$ \\
\hline 1 & $\begin{array}{l}\text { Reference Signal: washing machine noise } \\
\text { Primary Signal : recorded female speech signal }+ \\
\text { washing machine noise }\end{array}$ & $12.7318 \mathrm{~dB}$ & $29.3123 \mathrm{~dB}$ \\
\hline
\end{tabular}

Table 7: SNR Analysis (Signal Length:Time_in_sec $=27.4460 \mathrm{sec}$ ) 


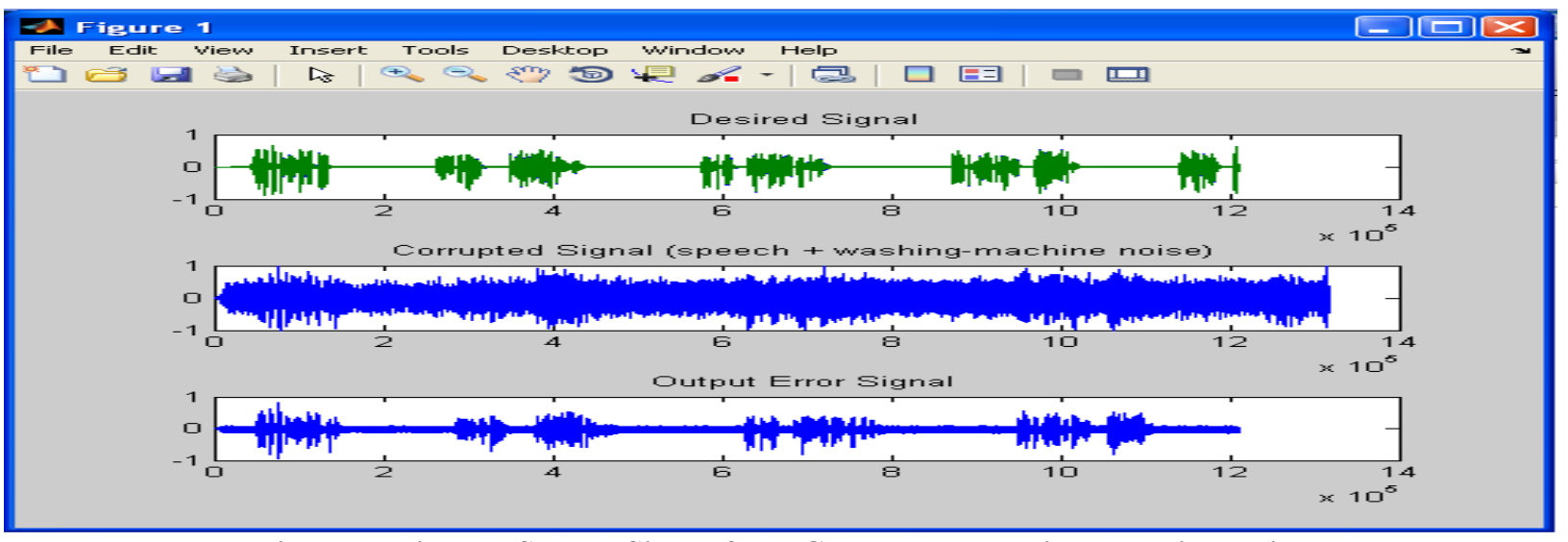

Figure 7: Filtered Speech Signal from Corrupted Washing Machine Noise

Thus, the system is tested for different noise signals. The five different experiments are implemented and respective results are presented. It can be concluded that the original and recovered signal obtained remains unchanged. The input and output Signal to noise ratio is measured by taking optimal parameters for analysis and is shown in Table 2, 3, 5,6 and 7. Further, it is observed that when the frequency of noise \& signal are very much similar in that case noise becomes more effective than main signal.

Thus, it can be concluded that as the step size value increases within the stability region, it increases convergence speed and also increases the mis-adjustment. And as the step size value decreases, it increases the convergence time and reduces the mis-adjustments. However, if we increase the filter order value, it increases the convergence time and also increases the misadjustments. Thus, LMS worked successfully to handle the noise related problems. This research will pave way to undertake scientific research in similar direction. Further the interested academicians can carry out further studies to enhance the performance of adaptive filters as extension of this research by using different approaches.

\section{Conclusion}

From the results obtained, it can be concluded that the proposed ANC system has been successfully implemented on MATLAB by considering more realistic noises. The results are analyzed for different input noises originating from various disturbances such as construction site, air conditionings, refrigerators, barking of dogs, vaccum cleaners as reference signals and human voice signal as primary signal. These signals are analyzed and the filter performance is measured in terms of SNR improvement.

It is evident from the results that SNR is clearly improved vis-à-vis filter performance. The speech signal is examined for different frequencies \& voltage level to determine its capabilities of noise filtering. The first experiment is based on human voice where female speech is considered as original signal contaminated with vacuum cleaner noise signal recorded for different time durations. The second experiment is based on female speech signal contaminated with the vaccum cleaner noise recorded at $\mathrm{t} 2$ and the reference signal i.e. undesired vaccum cleaner noise with low speech recorded at $\mathrm{t} 1$. From the experimentation it is clear that SNR of primary signal earlier it was $3.468 \mathrm{db}$ which gets substantially improved by $49.921 \mathrm{~dB}$. Subsequent experiments also shows similar improvement in signal to noise ratio measured by considering more realistic noise signals. It has also been observed that for filter order $\mathrm{N}=50$ and for convergence factor $\mu=0.03$, the voice signal can still be recovered perfectly. Based on these results, the designed system can effectively resolve the problem of unwanted noise and proved to be successful.

\section{References}

[1] George S. Kang, Member, IEEE, Lawrence J. Fransen,"Experimentation with an Adaptive Noise-Cancellation Filter," IEEE Transactions On Circuits and Systems, Vol. CAS-34, No. 7, July 1987, Pp. 753- 758.

[2] Vakulabharanam Ramakrishna, Tipparti Anil Kumar, "Low Power VLSI Implementation of Adaptive Noise Canceller Based on Least Mean Square Algorithm", 2013 4th International Conference on Intelligent Systems, Modeling and Simulation, 2166-0662/13, ISMS-2013, IEEE DOI 10.1109/ISMS.2013.84, pp. 276-279.

[3] Farzad Nekouei, Neda Zargar Talebi, Yousef S. Kavian, Ali Mahani, "FPGA Implementation of LMS Self Correcting Adaptive Filter (SCAF) and Hardware Analysis", $8^{\text {th }}$ IEEE, IET International Sympoaium on Communication Systems, Networks and Digital Signal Processing, 978-1-4577-1473-3/12, 2012 IEEE. 
[4] Hong-Yuan Jheng, Yen-Hsiang Chen, Shanq-Jang Ruan and Ziming Qi, "FPGA Implementation of High Sampling Rate In-Car Non-Stationary Noise Cancellation Based on Adaptive Wiener Filter", 2011 IEEE/IFIP 19th International Conference on VLSI and System- on-Chip.

[5] Wolfgang Fohl, and Jörn Matthies, "An fpga-based adaptive noise cancelling system," Proceeding of the $12^{\text {th }}$ International Conference on Digital Audio Effects (DAFX), Como, Italy, September 2009, pp. 17.

[6] Ahmed Elhossini, Shawki Areibi, and Robert Dony, "An fpga implementation of the lms adaptive filter for audio processing," IEEE International Conference on Reconfigurable Computing and FPGA's, September 2006, pp. 1-8.

[7] Joonwan Kim, and A. D. Poularikas, "Comparison of two proposed methods in adaptive noise canceling," Proceedings of the $35^{\text {th }}$ Southeastern symposium on system theory (SSST), March 2003, pp.400-403.

[8] Rosado-Muñoz, A, Bataller-Mompe n, M. ; Soria-Olivas, E. ; Scarante, C. ; Guerrero-Martínez, J.F. "FPGA Implementation of an Adaptive Filter Robust to Impulsive Noise: Two Approaches," IEEE Transactions on Industrial Electronics, Volume 58, issue 3, March 2011, PP. 860-870.

[9] M. Bahoura, H. Ezzaidi, "FPGA-implementation of a sequential adaptive noise canceller using Xilinx System Generator," Proceedings of the 2009 International Conference on Microelectronics (ICM), pp. 213-216.

[10] Ma Shengqian ; Coll. of Phys. \& Electron. Eng., Northwest Normal Univ., Lanzhou, China ; Xu Guowei ; Ma Zhifeng ; Wei Shuping "Research on adaptive noise canceller of an improvement LMS algorithm," Electronics, Communications and Control (ICECC), 2011 International Conference on 9-11Sept. 2011, Print ISBN:978-1-4577-0320-1, IEEE, Zhejiang, DOI:10.1109/ICECC.2011.6067870, Page(s):1611 - 1614.

[11] S.S.Godbole, Dr. S. B. Pokle, "Implementation of Adaptive Noise Canceller in Digital Filter for Various Applications," CiiT International Journal of Digital Signal Processing, Coimbatore Institute of Information Tech., Coimbatore-641009. Tamil Nadu, India, 2011.

[12] Swati S. Godbole, Dr. S. B. Pokle, "Implementation of Adaptive Noise Canceller System for Audio related Applications," published in International Journal of Measurement Technologies and Instrumentation Engineering (IJMTIE-2014), 3(4), US, 51-67, DOI: 10.4018/ijmtie.2013100105, ISSN: 2156-1737, October-December 2013.

[13] Swati S. Godbole, Dr. S. B. Pokle, "Hardware Implementation Of Adaptive $\quad$ Filter for Noise Cancellation Using TMS320C6713 DSP," published in International Journal of Measurement Science and Instrumentation, JMSI-2014, China, vol. 5 No.3, DOI: 10.3969/j.issn.1674-8042.2014.03.008, CLD number: TN911.7, Article ID: 16748042(2014)03-0038-010, Sept. 2014.

[14] Haykin, Simon S. “Adaptive Filter Theory,” Upper Saddle River (N.J.): Prentice Hall, 2002, Print.

[15] Bernard Widrow and Samuel D. Steams "Adaptive Signal Processing," Prentice-Hall, Inc.,Englewood Cliffs, New Jersey, 1985.

[16] Widrow, B., J.R. Glover, J.M. McCool, J. Kaunitz, C.S.Williams, R.H. Hearn, J.R. Zeidler, Jr. Eugene Dong and R.C. Goodlin. "Adaptive Noise Cancelling: Principles and Applications," Proceedings of the IEEE 63.12 (1975): 1692-716, Print. 